\title{
PERIODIC ORBITS OF CONTINUOUS AND DISCONTINUOUS PIECEWISE LINEAR DIFFERENTIAL SYSTEMS VIA FIRST INTEGRALS
}

\author{
JAUME LLIBRE ${ }^{1}$ AND MARCO ANTONIO TEIXEIRA ${ }^{2}$
}

\begin{abstract}
The continuous or discontinuous piecewise linear differential systems are completely integrable in every piece. Using the first integrals of these differential systems we illustrate for two of such systems, one in $\mathbb{R}^{3}$ and the other in $\mathbb{R}^{4}$, how to study analytically their periodic solutions.
\end{abstract}

\section{InTRODUCTION AND STATEMENT OF THE MAIN RESUlt}

1.1. The goal. This paper is part of a general program involving the study of continuous and discontinuous piecewise differential systems in $\mathbb{R}^{n}$ of the form

$$
\dot{u}=f(u)+\operatorname{sign}\left(u_{1}\right) g(u),
$$

where $u=\left(u_{1}, \ldots, u_{n}\right)$, and $f, g: \mathbb{R}^{n} \rightarrow \mathbb{R}^{n}$ are smooth functions. Note that we have two different differential systems, one in the half-space $u_{1}>0$ and the other in $u_{1}<0$. Eventually on the hyperplane $u_{1}=0$ the piecewise differential system can be continuous or discontinuous.

Our main concern is to discuss the existence and robustness of periodic orbits for such piecewise differential systems assuming that both differential systems, the one in $u_{1}>0$ and the other in $u_{1}<0$, have first integrals.

We point out that, as far as we know, there is no developed theory of singularity of mappings (Morse Theory or similar) treating such objects.

1.2. Historical facts and motivations. Control Theory is a natural source of mathematical models of these systems (see, for instance, [?]). It is worth mentioning that Anosov in [2] studied a class of relay systems in $\mathbb{R}^{n}$ of the form

$$
\dot{u}=A u+\operatorname{sign}\left(u_{1}\right) k
$$

2010 Mathematics Subject Classification. 34C29, 34C25, 47H11.

Key words and phrases. periodic solutions, periodic orbits, continuous piecewise linear differential systems, first integrals, discontinuous piecewise differential systems. 
where $A$ is an $n \times n$ real matrix and $k=\left(k_{1}, \ldots, k_{n}\right)$ is a constant vector in $\mathbb{R}^{n}$.

Jacquemard and Teixeira in [7] analyzed the simplest model of a relay system $\dddot{x}=-\operatorname{sign}(x)$. The focus was to detect the existence and robustness of a one-parameter families of periodic orbits through reversible polynomial perturbations.

It is worthwhile to cite Ekeland [4] and Klok [8], where the main problem in the classical calculus of variations was carried out to study discontinuous Hamiltonian vector fields.

1.3. Integrability for smooth systems. Consider $C^{k}$ differential systems of the form

$$
\dot{x}=f(x), \quad x \in \Omega \subset \mathbb{R}^{n},
$$

where $k \in \mathbb{N} \cup\{\infty, \omega\}$, the dot denotes derivative with respect to the independent variable $t, \Omega$ is an open subset of $\mathbb{R}^{n}$, and $f(x)=$ $\left(f_{1}(x), \ldots, f_{n}(x)\right)$ is a $C^{k}$ function defined in $\Omega$. As usual $\mathbb{N}$ denotes the set of positive integers, and $C^{\infty}$ and $C^{\omega}$ denote the sets of infinitely derivable functions and analytic functions, respectively.

A first integral of system (1) is a continuous function $H(x)$ defined in a full Lebesgue measure subset $\Omega_{1}$ of $\Omega$, which is not locally constant on any positive Lebesgue measure subset of $\Omega_{1}$; moreover $H(x)$ is constant along each orbit of system (2) in $\Omega_{1}$. If $H(x)$ is $C^{1}$ then it is a first integral if and only if it satisfies

$$
\frac{\partial H}{\partial x_{1}} f_{1}(x)+\ldots+\frac{\partial H}{\partial x_{n}} f_{n}(x)=0,
$$

for all the points $x$ where $H(x)$ is defined.

System (2) is $C^{r}$ completely integrable in $\Omega$, if it has $n-1$ functionally independent $C^{r}$ first integrals in $\Omega$. The functions $H_{1}(x), \ldots, H_{k}(x)$ are functionally independent in $\Omega$ if their gradients $\nabla H_{1}, \ldots, \nabla H_{k}$ have rank $k$ in a full Lebesgue measure subset of $\Omega$.

The easiest class of completely integrable systems are the linear differential systems in $\mathbb{R}^{n}$, i.e. the differential systems of the form

$$
\dot{x}=A x+b,
$$

where $x, b \in \mathbb{R}^{n}$ and $A$ is an $n \times n$ real matrix. Clearly the domain of definition $\Omega$ of a linear differential system is the whole $\mathbb{R}^{n}$. In fact, every linear differential system is completely integrable with Darboux first integrals. Here a first integral is Darboux if it is a real function which can be written in the form

$$
f_{1}(x)^{\lambda_{1}} \cdots f_{k}(x)^{\lambda_{k}} e^{g(x) / h(x)},
$$


where $f_{i}(x)$ for $i=1, \ldots, k, g(x)$ and $h(x)$ are complex polynomials, and the $\lambda_{i}$ for $i=1, \ldots, k$ are complex numbers. For more details on Darboux first integrals see, for instance, $[5,11]$.

1.4. Setting the problem. The study of the piecewise linear differential systems, continuous or discontinuous, mainly started with the book of Andronov, Vitt and Khaikin [1] and still continues receiving a big attention by researchers, because these systems appear in a natural way modeling processes in mechanics, electronics, economy, ..., see for more details the books $[3,12]$, and the hundreds of references quoted therein.

To study analytically the periodic solutions of a differential system is a very difficult task, usually impossible of doing. Our goal here is to show that the periodic orbits of piecewise differential systems, continuous or discontinuous, which are completely integrable in each piece, "sometimes" can be studied analytically using the first integrals of these systems. This "sometimes" means that for such differential systems in theory always can be studied their periodic orbits using the first integrals, but in the practice the computations that must be done prevent frequently of doing this study.

More precisely, in this work we study analytically the periodic orbits of the following two discontinuous piecewise linear differential systems:

$$
\begin{aligned}
& \dot{x}=y, \\
& \dot{y}=z, \\
& \dot{z}=-\operatorname{sign}(x) y,
\end{aligned}
$$

and

$$
\begin{aligned}
& \dot{x}=y, \\
& \dot{y}=z, \\
& \dot{z}=u, \\
& \dot{u}=\operatorname{sign}(x),
\end{aligned}
$$

using their first integrals. As usually the sign function is defined as follows

$$
\operatorname{sign}(x)=\left\{\begin{array}{cl}
-1 & \text { if } x<0 \\
0 & \text { if } x=0 \\
1 & \text { if } x>0
\end{array}\right.
$$

So $x=0$ is the plane or hyperplane of discontinuity of the piecewise linear differential systems (2) or (3), respectively. Hence, both discontinuous piecewise linear differential systems are formed by two linear differential systems, one defined in the half-space $x \geq 0$ and the other in the half-space $x \leq 0$. 
The discontinuous piecewise linear differential systems (3) is the basic model of a semi-linear vector field see the book [13] for some properties of these systems.

If the discontinuous piecewise linear differential systems (2) or (3) have a periodic solution which cross two times the plane or hyperplane of discontinuity, then in its neighborhood it is defined a Poincaré return map. Then the usual way of studying the periodic solutions of these differential systems is looking for the fixed points of such Poincaré return map. Usually this is done splitting the Poincaré return map as the composition of two Poincaré maps, one from $x=0$ to itself defined only in the half-space $x \geq 0$, and the other from $x=0$ to itself defined only in the half-space $x \leq 0$. For studying each one of these two Poincaré maps we must compute the solutions of the linear differential systems in $x \geq 0$ and in $x \leq 0$, with initial conditions a point $p$ in $x=0$, and compute the times that these two solutions need for reaching again the plane or hyperplane $x=0$ by first time, for one solution in forward time and for the other in backward time. Once these two times are known, we must impose that the two solutions starting at the point $p$ of $x=0$ reached by first a same point of $x=0$. The problem for applying this algorithm usually comes from the fact that we cannot compute explicitly the required times. For illustrating the described algorithm see for instance [10].

There are other ways for computing the periodic solutions which cross two times the plane or hyperplane of discontinuity. Thus the recent developments of the averaging theory for discontinuous differential systems allow to study these periodic solutions, but again for applying this theory we need to know some explicit values of the independent variable of the system, which sometimes are not possible to compute. See for more details [9].

The goal of this work is to study the periodic solutions which cross two times the plane or hyperplane of discontinuity of the discontinuous piecewise linear differential systems (2) and (3) using the first integrals of their linear differential systems. As we said before this way of computing the mentioned periodic solutions also have frequently problems of computation. In any case we shall see that sometimes it works very well, as we shall show in the study of the periodic solutions of the two discontinuous piecewise linear differential systems (2) and (3). We remark that the study of the existence or non-existence of the periodic solutions of system (3) for the other two methods here mentioned (Poincaré return map or averaging theory) is extremely difficult. 
We have chosen discontinuous piecewise linear differential systems for illustrating how to use the first integrals for computing periodic solutions, but the same technique can be used for continuous piecewise linear differential systems.

Our main results are the following two theorems.

Theorem 1. Every periodic orbit of the discontinuous piecewise linear differential system (2) which intersects the discontinuous plane $x=0$ in two points, does it at the points $(0, y, z)$ and $(0,-y, z)$, for all $y$ and $z$ satisfying $z>0$ and $y^{2}-z^{2}<0$.

Theorem 1 is proved in section 2 . The proof of Theorem 1 only uses the first integrals of this system.

Theorem 2. Every periodic orbit of the discontinuous piecewise linear differential system (3) which intersects the discontinuous hyperplane $x=0$ in two points, does it at the points $\left(0,-u^{3} / 3,0, u\right)$ and $\left(0, u^{3} / 3,0,-u\right)$ for all $u \neq 0$.

Theorem 2 is proved in section 3. The proof of Theorem 2 uses the first integrals of the system and the fact that we know explicitly in function of the time the solutions of a linear differential system.

\section{Proof of Theorem 1}

The discontinuous piecewise linear differential system (2) in $\mathbb{R}^{3}$ is formed by the following two linear differential systems

$$
\begin{aligned}
& \dot{x}=y, \\
& \dot{y}=z, \\
& \dot{z}=-y,
\end{aligned}
$$

in the half-space $x>0$, and

$$
\begin{aligned}
& \dot{x}=y, \\
& \dot{y}=z, \\
& \dot{z}=y,
\end{aligned}
$$

in the half-space $x<0$. Note that both systems have the points of the $x$-axis contained in the half-spaces where they are defined as equilibrium points.

Since both differential systems are linear we always can compute two independent first integrals for each system, see for instance [5]. Thus two independent first integrals for system (4) are

$$
H_{1}=x+z, \quad \text { and } H_{2}=y^{2}+z^{2} .
$$


So the orbits of system (4) are contained in the sets

$$
\gamma_{h_{1} h_{2}}=\left\{H_{1}=h_{1}\right\} \cap\left\{H_{2}=h_{2}\right\} \cap\{x>0\},
$$

for all $\left(h_{1}, h_{2}\right) \in \mathbb{R}^{2}$ when these sets are non-empty.

In a similar way two independent first integrals for system (5) are

$$
F_{1}=x-z, \text { and } F_{2}=y^{2}-z^{2} .
$$

So the orbits of system (5) are contained in the sets

$$
\gamma_{f_{1} f_{2}}=\left\{F_{1}=f_{1}\right\} \cap\left\{F_{2}=f_{2}\right\} \cap\{x<0\},
$$

for all $\left(f_{1}, f_{2}\right) \in \mathbb{R}^{2}$ when these sets are non-empty.

We note that the set $\gamma_{h_{1} h_{2}}$, when it is non-empty and $h_{2}>0$, is formed by the piece of the connected curve obtained from the intersection of the plane $H_{1}=h_{1}$ with the cylinder $H_{2}=h_{2}$ and with the half-space $x>0$. So under these assumptions the set $\gamma_{h_{1} h_{2}}$ is a connected arc contained in $x>0$ which does not contain equilibria, so it is an orbit of system (4). If $\gamma_{h_{1} h_{2}}$ is non-empty and $h_{2}=0$, then $\gamma_{h_{1} h_{2}}$ is an equilibrium point. In short, always that the set $\gamma_{h_{1} h_{2}}$ is non-empty it is formed by a unique orbit of system (4).

It is easy to check that the set $\gamma_{f_{1} f_{2}}$ is always non-empty. If $f_{2} \neq 0$, is formed by one or two arcs of the curve obtained from the intersection of the plane $F_{1}=f_{1}$ with the hyperboloid of two sheets $F_{2}=f_{2}$ contained in the half-space $x<0$. Moreover these one or two arcs do not contain equilibria (because the equilibria need that $f_{2}=0$ ), so $\gamma_{f_{1} f_{2}}$ is formed by one or two orbits of system (5). If $f_{2}=0$, then $\gamma_{f_{1} f_{2}}$ is the intersection of the plane $F_{1}=f_{1}$ with the two planes $F_{2}=0$ and with the half-space $x<0$. The intersection $\left\{F_{1}=f_{1}\right\} \cap\left\{F_{2}=0\right\}$ is formed by two straight lines intersecting at the equilibrium point $\left(f_{1}, 0,0\right)$. The intersection of these two straight lines with the half-space $x<0$, can contain either 5 orbits (one of them is the equilibrium point $\left(f_{1}, 0,0\right)$ ), or 2 orbits.

We want to study when an orbit of $\gamma_{h_{1} h_{2}}$ and an orbit of $\gamma_{f_{1} f_{2}}$ can connect forming a periodic orbit of the discontinuous piecewise linear differential system (2), i.e. when the two orbits reach the plane $x=0$ in the same two points. In such a case they form a periodic solution because from system (2) these two points are crossing points. More precisely, let $X_{+}$(resp. $X_{-}$) be the vector field associated to the linear differential system (4) (resp. (5)) in the half-space $x \geq 0$ (resp. $x \leq 0$ ). Let $p$ be a point of the discontinuity plane $x=0$, when the segment connecting the endpoints of the vectors $X_{+}(p)$ and $X_{-}(p)$ does not intersect the plane $x=0$, then $p$ is a crossing point. For more details on crossing points see [6]. 
From the previous study done on the orbits of systems (4) and (5) it follows that an orbit of $\gamma_{h_{1} h_{2}}$ and an orbit of $\gamma_{f_{1} f_{2}}$ can connect forming a periodic orbit only if $h_{2}>0$ and $f_{2} \neq 0$.

We take an arbitrary point of the plane of discontinuity, for instance the point $\left(0, y_{0}, z_{0}\right)$. We evaluate the four first integrals $H_{1}, H_{2}, F_{1}$ and $F_{2}$ in this point and we get the four values $h_{1}=z_{0}, h_{2}=y_{0}^{2}+z_{0}^{2}$, $f_{1}=-z_{0}$ and $f_{2}=y_{0}^{2}-z_{0}^{2}$, respectively. Now we study how many points of the orbit $\left\{H_{1}=h_{1}\right\} \cap\left\{H_{2}=h_{2}\right\} \cap\{x \geq 0\}$ are in the plane $x=0$, solving the system

$$
H_{1}=h_{1}, \quad H_{2}=h_{2}, \quad x=0,
$$

we get two points $\left(0, \pm y_{0}, z_{0}\right)$. We also analyze how many points of the orbits $\left\{F_{1}=f_{1}\right\} \cap\left\{F_{2}=f_{2}\right\} \cap\{x \leq 0\}$ are in the plane $x=0$, solving the system

$$
F_{1}=f_{1}, \quad F_{2}=f_{2}, \quad x=0,
$$

again we get the two points $\left(0, \pm y_{0}, z_{0}\right)$. If these two points belong to the same orbit of the set $\left\{F_{1}=f_{1}\right\} \cap\left\{F_{2}=f_{2}\right\} \cap\{x \leq 0\}$, then we have a periodic orbit of the discontinuous piecewise linear differential system (2).

If we parameterize the orbit $\left\{H_{1}=h_{1}\right\} \cap\left\{H_{2}=h_{2}\right\}$ in the half-space $x \geq 0$ using the variable $x$ we obtain the arc

$$
\left\{\left(x, \pm \sqrt{y_{0}^{2}+z_{0}^{2}-\left(z_{0}-x\right)^{2}}, z_{0}-x\right): 0 \leq x \leq z_{0}+\sqrt{y_{0}^{2}+z_{0}^{2}}\right\} .
$$

Note that this orbit is symmetric with respect to the $y$-axis, it is contained in $x \geq 0$ and its endpoints are the two points $\left(0, \pm y_{0}, z_{0}\right)$ on the plane $x=0$.

Again we parameterize the curve $\left\{F_{1}=f_{1}\right\} \cap\left\{F_{2}=f_{2}\right\}$ in the half-space $x \leq 0$ using the variable $x$. This curve is formed by

- the two orbits $\left\{\left(x, \sqrt{x^{2}+y_{0}^{2}}, x\right): x \leq 0\right\}$ and $\left\{\left(x,-\sqrt{x^{2}+y_{0}^{2}}, x\right)\right.$ : $x \leq 0\}$ if $z_{0}=0$, each orbit has only one endpoint in the plane $x=0$;

- the two orbits $\left.\left.\left\{x, \sqrt{\left(x+z_{0}\right)^{2}+y_{0}^{2}-z_{0}^{2}}, z_{0}+x\right)\right\}: x \leq 0\right\}$ and $\left.\left.\left\{x,-\sqrt{\left(x+z_{0}\right)^{2}+y_{0}^{2}-z_{0}^{2}}, z_{0}+x\right)\right\}: x \leq 0\right\}$ if either $z_{0}<0$ or $z_{0}>0$ and $y_{0}^{2}-z_{0}^{2}>0$, each orbit has only one endpoint in the plane $x=0$;

- the two orbits

$\left.\left.\left\{x, \pm \sqrt{\left(x+z_{0}\right)^{2}+y_{0}^{2}-z_{0}^{2}}, z_{0}+x\right)\right\}:-z_{0}+\sqrt{z_{0}^{2}-y_{0}^{2}} \leq x \leq 0\right\}$ 
and

$$
\left.\left.\left\{x, \pm \sqrt{\left(x+z_{0}\right)^{2}+y_{0}^{2}-z_{0}^{2}}, z_{0}+x\right)\right\}: x \leq-z_{0}-\sqrt{z_{0}^{2}-y_{0}^{2}}\right\}
$$

if $z_{0}>0$ and $y_{0}^{2}-z_{0}^{2}<0$, the first orbit has its two endpoints at the points $\left(0, \pm y_{0}, z_{0}\right)$ of the plane $x=0$, and the second orbit has its endpoints at infinity;

We note that $y_{0}^{2}-z_{0}^{2} \neq 0$ otherwise $f_{2}=0$.

In short, if $z_{0}>0$ and $y_{0}^{2}-z_{0}^{2}<0$ then the orbit (6) of the linear differential system (4) together with the orbit (7) of the linear differential system (5) form a periodic orbit of the discontinuous piecewise linear differential system (2), and this periodic orbit intersect the plane of discontinuity $x=0$ at the two points $\left(0, \pm y_{0}, z_{0}\right)$. This completes the proof of Theorem 1 .

\section{Proof of Theorem 2}

The discontinuous piecewise linear differential system (3) in $\mathbb{R}^{4}$ is formed by the following two linear differential systems

$$
\begin{aligned}
& \dot{x}=y, \\
& \dot{y}=z, \\
& \dot{z}=u, \\
& \dot{u}=1,
\end{aligned}
$$

in the half-space $x>0$, and

$$
\begin{aligned}
& \dot{x}=y, \\
& \dot{y}=z, \\
& \dot{z}=u, \\
& \dot{u}=-1,
\end{aligned}
$$

in the half-space $x<0$. Note that both systems have no equilibrium points.

Since both differential systems are linear we always can compute three independent first integrals for each system, see for instance [5]. Thus three independent first integrals for system (8) are

$$
H_{1}=8 x-8 y u+4 z u^{2}-u^{4}, \quad H_{2}=3 y-3 z u+u^{3}, \quad H_{3}=2 z-u^{2} .
$$

So the orbits of system (4) are contained in the sets

$$
\gamma_{h_{1} h_{2} h_{3}}=\left\{H_{1}=h_{1}\right\} \cap\left\{H_{2}=h_{2}\right\} \cap\left\{H_{3}=h_{3}\right\} \cap\{x>0\},
$$

for all $\left(h_{1}, h_{2}, h_{3}\right) \in \mathbb{R}^{3}$ when these sets are non-empty.

In a similar way three independent first integrals for system (9) are

$$
F_{1}=8 x+8 y u+4 z u^{2}+u^{4}, \quad F_{2}=3 y+3 z u+u^{3}, \quad F_{3}=2 z+u^{2} .
$$


So the orbits of system (5) are contained in the sets

$$
\gamma_{f_{1} f_{2}, f_{3}}=\left\{F_{1}=f_{1}\right\} \cap\left\{F_{2}=f_{2}\right\} \cap\left\{F_{3}=f_{3}\right\} \cap\{x<0\},
$$

for all $\left(f_{1}, f_{2}, f_{3}\right) \in \mathbb{R}^{3}$ when these sets are non-empty.

Again we are looking for the periodic orbits of the discontinuous piecewise linear differential system (3) which have two points on the hyperplane of discontinuity $x=0$. Let $\left(0, y_{0}, z_{0}, u_{0}\right)$ one of these two points. We evaluate the six first integrals $H_{1}, H_{2}, H_{3} F_{1}, F_{2}$ and $F_{3}$ in this point and we get the six values $h_{1}=-8 y_{0} u_{0}+4 z_{0} u_{0}^{2}-u_{0}^{4}$, $h_{2}=3 y_{0}-3 z_{0} u_{0}+u_{0}^{3}, h_{3}=2 z_{0}-u_{0}^{2}, f_{1}=8 y_{0} u_{0}+4 z_{0} u_{0}^{2}+u_{0}^{4}, f_{2}=$ $3 y_{0}+3 z_{0} u_{0}+u_{0}^{3}$ and $f_{3}=2 z_{0}+u_{0}^{2}$, respectively. Now we study how many points of the set $\gamma_{h_{1} h_{2} h_{3}} \cap \gamma_{f_{1} f_{2}, f_{3}}$ are in the hyperplane $x=0$. Of course, if the system has periodic orbits having two points in the hyperplane $x=0$, there must exist sets set $\gamma_{h_{1} h_{2} h_{3}} \cap \gamma_{f_{1} f_{2}, f_{3}}$ having at least two points in the hyperplane $x=0$. We claim that all the sets $\gamma_{h_{1} h_{2} h_{3}} \cap \gamma_{f_{1} f_{2}, f_{3}}$ have exactly two points in the hyperplane $x=0$ if $u_{0} \neq 0$, the points $\left(0, y_{0}, z_{0}, u_{0}\right)$ and $\left(0,-y_{0}, z_{0},-u_{0}\right)$; if $u_{0}=0$ the sets $\gamma_{h_{1} h_{2} h_{3}} \cap \gamma_{f_{1} f_{2}, f_{3}}$ have a unique point in the hyperplane $x=0$, the point $\left(0, y_{0}, z_{0}, 0\right)$. So when $u_{0}=0$ the discontinuous piecewise linear differential system (3) has no periodic solutions through the point $\left(0, y_{0}, z_{0}, 0\right)$.

Now we shall prove the claim. The points of the set $\gamma_{h_{1} h_{2} h_{3}} \cap \gamma_{f_{1} f_{2}, f_{3}}$ which are on the hyperplane $x=0$ are the solutions of the system

$$
H_{k}=h_{k}, \quad F_{k}=f_{k}, \quad \text { for } k=1,2,3,
$$

restricted to $x=0$, i.e. we must solve the system

$$
\begin{array}{ll}
8 x-8 y u+4 z u^{2}-u^{4} & =-8 y_{0} u_{0}+4 z_{0} u_{0}^{2}-u_{0}^{4}, \\
3 y-3 z u+u^{3} & =3 y_{0}-3 z_{0} u_{0}+u_{0}^{3}, \\
2 z-u^{2} & =2 z_{0}-u_{0}^{2} \\
8 x+8 y u+4 z u^{2}+u^{4} & =8 y_{0} u_{0}+4 z_{0} u_{0}^{2}+u_{0}^{4}, \\
3 y+3 z u+u^{3} & =3 y_{0}+3 z_{0} u_{0}+u_{0}^{3}, \\
2 z+u^{2} & =2 z_{0}+u_{0}^{2} .
\end{array}
$$

Adding the third and the sixth equations we get that $z=z_{0}$. Then from the third we get $u= \pm u_{0}$. Now assume $u=u_{0}$, then from the second it follows that $y=y_{0}$. Finally from the first we obtain that $x=0$. Therefore we get the known solution $(x, y, z, u)=\left(0, y_{0}, z_{0}, u_{0}\right)$. Consider now $u=-u_{0} \neq 0$, then system (10) reduces to

$$
\begin{array}{ll}
8 x+8 y u_{0} & =-8 y_{0} u_{0}, \\
3 y+3 z_{0} u_{0}-u_{0}^{3} & =3 y_{0}-3 z_{0} u_{0}+u_{0}^{3}, \\
8 x-8 y u_{0} & =8 y_{0} u_{0}, \\
3 y-3 z_{0} u_{0}-u_{0}^{3} & =3 y_{0}+3 z_{0} u_{0}+u_{0}^{3} .
\end{array}
$$


Adding the first and the third equation we get $x=0$. Then from the first we get $y=-y_{0}$. So we have obtained the solution $(x, y, z, u)=$ $\left(0,-y_{0}, z_{0},-u_{0}\right)$. Finally assume that $u=u_{0}=0$, then system (10) becomes

$$
\begin{aligned}
& 8 x=0, \\
& 3 y=3 y_{0}, \\
& 8 x=0 \\
& 3 y=3 y_{0} .
\end{aligned}
$$

So system (10) has a unique solution $(x, y, z, u)=\left(0, y_{0}, z_{0}, 0\right)$. Hence the claim is proved.

In short, we have proved that if the discontinuous piecewise linear differential system (3) has some periodic orbit intersecting the hyperplane $x=0$ in two points, these two points must be of the form $\left(0, y_{0}, z_{0}, u_{0}\right)$ and $\left(0,-y_{0}, z_{0},-u_{0}\right)$ with $u_{0} \neq 0$.

The solution of the linear differential system (8) starting at the point $\left(0, y_{0}, z_{0}, u_{0}\right)$ when $t=0$ is

$$
\begin{aligned}
& x_{+}(t)=\frac{1}{24}\left(t^{4}+4 u_{0} t^{3}+12 z_{0} t^{2}+24 y_{0} t\right), \\
& y_{+}(t)=\frac{1}{6}\left(t^{3}+3 u_{0} t^{2}+6 z_{0} t+6 y_{0}\right) \\
& z_{+}(t)=\frac{1}{2}\left(t^{2}+2 u_{0} t+2 z_{0}\right) \\
& u_{+}(t)=t+u_{0} .
\end{aligned}
$$

Similarly the solution of the linear differential system (9) starting at the point $\left(0, y_{0}, z_{0}, u_{0}\right)$ when $t=0$ is

$$
\begin{aligned}
& x_{-}(t)=\frac{1}{24}\left(-t^{4}+4 u_{0} t^{3}+12 z_{0} t^{2}+24 y_{0} t\right), \\
& y_{-}(t)=\frac{1}{6}\left(-t^{3}+3 u_{0} t^{2}+6 z_{0} t+6 y_{0}\right), \\
& z_{-}(t)=\frac{1}{2}\left(-t^{2}+2 u_{0} t+2 z_{0}\right), \\
& u_{-}(t)=-t+u_{0} .
\end{aligned}
$$

Assume that a periodic solution of the discontinuous piecewise linear differential system (3) exists passing through the two points $\left(0, y_{0}, z_{0}, u_{0}\right)$ and $\left(0,-y_{0}, z_{0},-u_{0}\right)$. Then we can define $t_{+}$the time that the solution $\left(x_{+}(t), y_{+}(t), z_{+}(t), u_{+}(t)\right)$ needs for reaching by first time the point $\left(0,-y_{0}, z_{0},-u_{0}\right)$ assuming that $x_{+}(t)>0$ in the interval of time with endpoints 0 and $t_{+}$. In a similar way let $t_{-}$be the time that the solution $\left(x_{-}(t), y_{-}(t), z_{-}(t), u_{-}(t)\right)$ needs for reaching by first time the 
point $\left(0,-y_{0}, z_{0},-u_{0}\right)$ assuming that $x_{-}(t)<0$ in the interval of time with endpoints 0 and $t_{-}$. Then we have

$$
\begin{array}{llll}
x_{+}\left(t_{+}\right)=0, & y_{+}\left(t_{+}\right)=-y_{0}, & z_{+}\left(t_{+}\right)=z_{0}, & u_{+}\left(t_{+}\right)=-u_{0} \\
x_{-}\left(t_{-}\right)=0, & y_{-}\left(t_{-}\right)=-y_{0}, & z_{-}\left(t_{-}\right)=z_{0}, & u_{-}\left(t_{-}\right)=-u_{0} .
\end{array}
$$

From the equation $u_{+}\left(t_{+}\right)=-u_{0}$ we get that $t_{+}=-2 u_{0}$. Then the equations $x_{+}\left(t_{+}\right)=0, y_{+}\left(t_{+}\right)=-y_{0}$ and $z_{+}\left(t_{+}\right)=z_{0}$ reduce to

$$
u_{0}^{4}-3 u_{0}^{2} z_{0}+3 u_{0} y_{0}=0, \quad u_{0}^{3}-3 u_{0} z_{0}+3 y_{0}=0 .
$$

Similarly from the equation $u_{-}\left(t_{-}\right)=-u_{0}$ we get that $t_{-}=2 u_{0}$. Then the equations $x_{-}\left(t_{-}\right)=0, y_{-}\left(t_{-}\right)=-y_{0}$ and $z_{-}\left(t_{-}\right)=z_{0}$ reduce to

$$
u_{0}^{4}+3 u_{0}^{2} z_{0}+3 u_{0} y_{0}=0, \quad u_{0}^{3}+3 u_{0} z_{0}+3 y_{0}=0 .
$$

Recall that $u_{0}$ cannot be zero. Then the solutions of system (11) and (12) with $u_{0} \neq 0$ are $y_{0}=-u_{0}^{3} / 3$ and $z_{0}=0$.

Summarizing if a periodic solution of the discontinuous piecewise linear differential system (3) exists having two points in the hyperplane $x=0$, these two points must be of the form $\left(0,-u_{0}^{3} / 3,0, u_{0}\right)$ and $\left(0, u_{0}^{3} / 3,0,-u_{0}\right)$ with $u_{0} \neq 0$. Then

$$
x_{+}(t)=\frac{1}{24}\left(t^{4}+4 u_{0} t^{3}-8 u_{0}^{3} t\right)
$$

and

$$
x_{-}(t)=\frac{1}{24}\left(-t^{4}+4 u_{0} t^{3}-8 u_{0}^{3} t\right) .
$$

The zeros of the polynomial $t^{4}+4 u_{0} t^{3}-8 u_{0}^{3} t$ in the variable $t$ are

$$
0, \quad-2 u_{0}, \quad-(1+\sqrt{5}) u_{0} \quad \text { and } \quad(\sqrt{5}-1) u_{0} .
$$

While zeros of the polynomial $-t^{4}+4 u_{0} t^{3}-8 u_{0}^{3} t$ in the variable $t$ are

$$
0, \quad 2 u_{0}, \quad(1+\sqrt{5}) u_{0} \text { and }(1-\sqrt{5}) u_{0} .
$$

Therefore it follows that $x_{+}(t)>0$ in the interval of time with endpoints 0 and $t_{+}$, and that $x_{-}(t)<0$ in the interval of time with endpoints 0 and $t_{-}$. This implies that the unique periodic solutions of the discontinuous piecewise linear differential system (3) having two points in the hyperplane of discontinuity $x=0$ are the ones passing through the two points $\left(0,-u_{0}^{3} / 3,0, u_{0}\right)$ and $\left(0, u_{0}^{3} / 3,0,-u_{0}\right)$ for all $u_{0} \neq 0$. This completes the proof of Theorem 2 . 


\section{ACKNOWLEDGMENTS}

The first author is partially supported by a MINECO/FEDER grant MTM2008-03437 and MTM2013-40998-P, an AGAUR grant number 2014SGR568, an ICREA Academia, the grants FP7-PEOPLE-2012IRSES 318999 and 316338, FEDER-UNAB-10-4E-378, and a CAPES grant 88881. 030454/2013-01 do Programa CSF-PVE. The second author is partially supported by ??????????

\section{REFERENCES}

[1] A. Andronov, A. Vitt and S. Khaikin, Theory of Oscillations, Pergamon Press, Oxford, 1966.

[2] D.V. Anosov, Stability of the equilibrium positions in relay systems, Automation and remote control XX, 2, (1959); auk SSSR 14 (1937), 247-250.

[3] M. di Bernardo, C.J. Budd, A.R. Champneys, P. Kowalczyk, Piecewise-Smooth Dynamical Systems: Theory and Applications, Appl. Math. Sci. Series 163, Springer-Verlag, London, 2008.

[4] I. Ekeland, Discontinuités de champs hamiltoniens et existence de solutions optimales en calcul des variations, IHES Publ. Math. 47 (1977), 5-32.

[5] M. FAlCONi AND J. LliBRe, $n-1$ independent first integrals for linear differential systems in $\mathbb{R}^{n}$ and $\mathbb{C}^{n}$, Qualitative Theory and Dynamical Systems 4 (2004), 233-254.

[6] A. F. Filippov, Differential Equations with Discontinuous Righthand Side, Mathematics and Its Applications, Kluwer Academic Publishers, Dordrecht, 1988.

[7] A. Jacquemard And M.A. Teixeira, Invariant varieties of discontinuous vector fields, Nonlinearity18 (2005), 21-43.

[8] F. KLOK, Broken solutions of homogeneous variational problems, J. Differential Equations 55 (1984), 101-134.

[9] J. Llibre, D.D. Novaes and M.A. Teixeira, On the birth of limit cycles for non-smooth dynamical systems, to appear in Bull. des Sciences Mathématiques, 2014.

[10] J. Llibre AND E. Ponce, Three nested limit cycles in discontinuous piecewise linear differential systems with two zones, Dynamics of Continuous, Discrete and Impulsive Systems, Serie B 19 (2012), 325-335.

[11] J. Llibre AND X. ZhANG, Darboux theory of integrability in $\mathbb{C}^{n}$ taking into account the multiplicity, J. of Differential Equations 246 (2009), 541-551.

[12] D.J.W. Simpson, Bifurcations in Piecewise-Smooth Continuous Systems, World Scientific Series on Nonlinear Science A, vol 69, World Scientific, Singapore, 2010

[13] M.I. Zelikin And V.F. Borisov, Theory of Chattering Control with Applications to Astronautics, Robotics, Economics, and Engineering, Birkhäuser, Bassel, 1994.

1 Departament de Matemàtiques, Universitat Autònoma de Barcelona, 08193 Bellaterra, Barcelona, Catalonia, Spain 
2 Departamento de Matemática, Universidade Estadual de Campinas, Caixa Postal 6065, 13083-970, Campinas, SP, Brazil

E-mail address: jllibre@mat.uab.cat

E-mail address: teixeira@ime.unicamp.br 